\title{
Establishing a Novel Algorithm for Highly Responsive Storage Space Allocation Based on NAR and Improved NSGA-III
}

\author{
Peijian Wu $(\mathbb{D}$ and Yulu Chen \\ School of Business and Administration, Anhui University of Finance and Economics, Bengbu 233030, China \\ Correspondence should be addressed to Yulu Chen; 3074085035@qq.com
}

Received 26 June 2021; Revised 2 February 2022; Accepted 5 February 2022; Published 24 February 2022

Academic Editor: Daniele Salvati

Copyright (๑) 2022 Peijian Wu and Yulu Chen. This is an open access article distributed under the Creative Commons Attribution License, which permits unrestricted use, distribution, and reproduction in any medium, provided the original work is properly cited.

\begin{abstract}
Establishing a rapid-response mechanism to manage customer orders is very important in managing demand surges. In this study, combined with predicting order requests, we established a multiobjective optimization model to solve the warehouse space allocation problem. First, we developed a model based on the NAR neural network to predict order requests. Subsequently, we used the improved NSGA-III based on good point set theory to construct a multiobjective optimization model to minimize resource loss, maximize efficiency in goods selection, and maximize goods accumulation. The following three modes were tested to allocate warehouse storage space: random, ABC, and prediction-oriented. Finally, using actual order data, we conducted a comparative analysis of the three modes regarding their efficiency in goods selection. The method proposed by this study improved goods selection efficiency by a sizable margin $(23.8 \%)$.
\end{abstract}

\section{Introduction}

As an essential part of logistics activities, warehousing and warehouse management play a vital role in manufacturing companies, third-party logistics companies, and distribution centers [1]. Moreover, with the advent of the big data era and the development of technologies such as the Internet of Things, business interconnection, and artificial intelligence, the flow of data for individual goods in a warehouse provides a massive source of data to predict future trends in the flow of goods and to take decisions about warehouse management $[2,3]$. At the same time, improving operational efficiency through the effective optimization of cargo space in warehouse operations results in increased savings. Effectively improving warehouse operations' efficiency has, therefore, become an industry-wide goal [4].

Storage space allocation is an essential part of the manufacturing industry's storage and transportation activities. In the manufacturing industry, storage space allocation and transportation play a decisive role in the level of efficiency with which goods enter and leave the warehouse. Therefore, a reasonable method of cargo space allocation can effectively shorten working time, improve efficiency, extend the shelf life, and reduce operating costs and is inseparable from the overall benefits of more efficient enterprises $[5,6]$. However, with the expansion of enterprise business scale and high responsive demand from customers, traditional space allocation methods such as the $\mathrm{ABC}$ classification method, random allocation, and storage strategy based on goods level fail to meet the current operational needs [7-9].

The logistics function is an important part of any business operations. From material procurement, organization of production, and customer sales to the hands of customers until to after-sales service, the flow of goods and services is indispensable. The accurate processing of big data can improve the accurate forecasting of market demand, flag when commodity inventories need replenishing, and even reduce equipment maintenance costs [10].

Accurate order prediction can provide a significant basis for future business growth by responding quickly to customer demand and coping with the changing market environment. Passive order management is not highly responsive toward this aspect, and it is challenging to meet the highly responsive needs of customers [11]. The massive 
time-series flow information generated by logistics and warehousing operations can support the decision for the management of demand in future periods. The warehouse space allocation combined with demand forecasting has more practical applications, value and research significance for enterprises.

Given that the current study focuses on goods demand prediction or goods allocation alone, the proposed models are based mostly on strict assumptions and lack the support of a large amount of data. This study combines the two problems and proposes the innovative use of NAR neural networks to predict time-series goods demand and then improves the NSGA-III algorithm by combining it with good point set theory to construct a highly responsive space allocation model.

\section{Literature Review}

The optimal storage space allocation involves multiple objectives, such as maximizing picking efficiency and minimizing machine travel distance $[12,13]$, while the storage depot contains most of the operational aspects of the logistics business process. Thus, it means that a variety of other factors such as the design of the storage area, the physical characteristics of the product, and the picking method need to be considered when performing storage space optimization $[14,15]$, so the storage space optimization problem is a multiobjective high-dimensional optimization problem.

Because of the complexity of the storage space allocation problem, the exact methods proposed by many scholars have not been executed to yield optimal solutions. The methods used contain mixed-integer programming $[9,16,17]$, robust optimization [18], binary programming [19], etc. Mixedinteger programming is the most used model by scholars, while robust optimization is the least implemented. Larco et al. [20] used a mixed-integer programming model with the optimization objective set to minimize order preparation time and employee discomfort. Bodnar and Lysgaard [21] used a dynamic planning algorithm to reduce the total amount of shelf replenishment in each period. Exact solution models have also been developed for specific types of warehouses. Ene et al. [22] used a class-based storage strategy to solve the warehouse stock allocation problem, obtained the optimal solution through an integer programming model, and developed an improved genetic algorithm to form optimal batches and routes for order picking.

To solve the constructed space allocation model, many scholars have studied, compared, and validated it using metaheuristic algorithms and their improved versions. Among them, genetic algorithms are the most widely used [22-24]. For example, Li et al. [8] defined a new dynamic storage space allocation problem based on $A B C$ classification and association between products, using genetic algorithms to deal with computational complexity and developing an integrated mechanism for optimization. Cruz-Dominguez and Santos-Mayorga [25] used a combination of genetic algorithms and artificial neural networks to minimize the order preparation for a given input condition considering the physical characteristics of the products and their sales patterns. Kim and Smith [26] used an improved simulated annealing algorithm to derive the algorithm's effectiveness to solve the space allocation problem based on data arithmetic examples of large distribution systems.

The current study rarely considers the demand forecasting of goods, and, at the same time, the assumptions of the constructed space allocation model do not meet the actual operational requirements of the enterprise. In the new management context of the big data era, enterprises' management decisions and optimization ideas must be future-oriented [27, 28]. Mezzogori and Zammori [29] discussed the application of deep learning two-layer architecture in fashion product demand forecasting, which after ten years of sales analysis has been proved; the forecasting method has advantages over the existing marketing strategies of fashion companies. Huber and Stuckenschmidt [30] focused on chain store order demand on particular dates, transforming the forecasting problem into supervised machine learning and evaluating multiple methods to conclude that the machine learning model has superior performance. $\mathrm{Xu}$ and Chan [31] used big data and machine learning methods to implement forecasting of medical device demand to establish a univariate device demand forecasting method, concluding that model prediction accuracy can be further improved by introducing big data into the forecasting model. Tarallo et al. [32] presented an exploratory study of machine learning methods for forecasting demand for products with a short shelf life and concluded that demand forecasting for FMCG products can improve the supply chain inventory balance and increase corporate profits.

A significant problem with the existing research is that the mathematical models developed relied on analytical models based on strict assumptions. However, some of these assumptions are not accurate and are not supported by massive data inputs [33, 34]. Current well-documented theoretical studies have shown that data generated from various industry sectors, when fully applied, will significantly improve management effectiveness and economic performance $[35,36]$. NAR artificial neural networks, with easily adjustable parameters, can provide demand forecasting with high accuracy and with improved NSGA-III based on the good points set theory, resulting in a highly responsive space allocation strategy. Using the improved NSGA-III and NAR demand forecasting can effectively solve warehouse space allocation problems, combined with demand prediction, providing the essential reference value for companies to improve operational efficiency and meet customers' growing needs.

\section{Research Method}

3.1. NAR Neural Network. An artificially generated neural network, which is established by simulating the human nervous system, can achieve specific functions. The network is constructed based on the structure connecting various neurons in the brain [37]. The NAR neural network uses itself as the regression variable and describes the random 
variable at a particular time in the future using a linear combination of variables from several moments identified during the observation period. The model's basic structure can be expressed by the following equation, where $e(n)$ represents the white noise that occurs during the data collection process. Further, this equation can be used to determine whether the observed value $y(n+1)$ at a particular moment has a certain correlation with the previous $n$ number of values [38].

$$
\begin{aligned}
y(t)= & a_{0}+a_{1} y(t-1)+a_{2} y(t-2)+a_{3} y(t-3) \\
& +\cdots+a_{n} y(t-n)+e(n) .
\end{aligned}
$$

Figure 1 depicts the structure of a NAR neural network. The network's input and output layers are determined by the background of the problem under study. The adjustment of the hidden layer and the number of neurons in the layer depends on the prediction results' accuracy [39]. During data learning and training, the NAR neural network discovers potential patterns and minimizes the difference between the final predicted outputs and actual records through a continuous and iterative fitting process [40].

3.2. Good Point Set Theory (GPS). GPS theory, proposed by Hua Luogeng, has superior properties: it can provide a theoretical basis for approximation calculations in higher dimensions, and the points selected by GPS have much more minor deviations than those randomly selected by the original NSGA-III algorithm.

Definition 1. Let $G_{s}$ be the unit cube in the s-dimension Euclidean space; we have $\langle x\rangle=\left(x_{1}, x_{2}, \ldots, x_{s}\right)$, where $0 \leqslant x_{i} \leqslant 1(i=1,2, \ldots, s)$.

Definition 2. The sample points $\langle r\rangle=\left(r_{1}, r_{2}, \ldots, r_{s}\right)=r_{1}$ $r_{2} \cdots r_{s}$ are compared with the target set $P_{n}(i)$ one by one, and $0 \leqslant x_{i} \leqslant 1(i=1,2, \ldots, s), \Phi(n)=\operatorname{Sup}\left|N_{n}(r) / n-\right|\langle r\rangle \|$; then the point set $P_{n}(i)$ is said to have deviation $\Phi(n)$, where $P_{n}(i)=\left(x_{1}^{(n)}(i), x_{2}^{(n)}(i), \quad \ldots, x_{s}^{(n)}(i)\right), 1 \leq i \leq n$, $x_{i}^{(n)}(i)=c\left(e^{j} \times i\right)(1<j<s) . c_{k}$ is the fractional part of $k$, and $N_{n}(\langle r\rangle)=N_{n}\left(r_{1}, r_{2}, \ldots, r_{s}\right)$ is the number of points in $P_{n}(i)$ that satisfy the condition $0 \leq x_{i}^{(n)}(i)<r_{j}$.

Definition 3. If $\Phi(n)$ satisfies $\Phi(n)=C(\langle r\rangle, \varepsilon) n^{-1+\varepsilon}$, where $C(\langle r\rangle, \varepsilon)$ is a constant related only to $\langle r\rangle$ and $\varepsilon$ ( $\varepsilon$ is an arbitrarily small positive number), then $P_{n}(i)$ is said to be a good point set (GPS) and $\langle r\rangle$ is called a good point. In addition, take $r_{k}=2 \cos 2 \pi k / p, 1 \leq k \leq s$, and $p$ is the smallest prime number satisfying $(p-s) / 2 \geq s$; then $\langle r\rangle$ is also a good point.

Theorem 1. Given that $P_{n}(i)(1 \leq i \leq n)$ has deviation $\Phi(n), f \in B_{t}$ (t-dimensional catch-all function class), then

$$
\left|\int_{x \in G} f(x) \mathrm{d} x-\sum \frac{f\left(P_{n}(i)\right)}{n}\right| \leq V(f) \Phi(n),
$$

whereV $(f)$ is the total variance of $f$.
Theorem 2. If $f(x)$ satisfies

$|f| \leq L,\left|\frac{\partial f}{\partial x_{i}}\right| \leq L, \ldots,\left|\frac{\partial^{2} f}{\partial x_{i} \partial x_{j}}\right| \leq L, \ldots,\left|\frac{\partial^{s} f}{\partial x_{i} \cdot \partial \partial x_{s}}\right| \leq L(1 \leq i \leq n)$.

then, using any weighting constituted by the value of the function given at $n$ points and using this approximation to calculate the integral of the function $f(x)$ over $G_{s}$, the error is not greater than $\mathrm{O}\left(n^{-1}\right)$.

Theorem 3. Assuming that $x_{1}, x_{2}, \ldots, x_{n}$ is uniformly distributed on $D_{t}$, that is, $P_{n}=\left\{x_{1}, x_{2}, \ldots, x_{n}\right\}$, then the deviation of $P_{n}$ is $D\left(n, P_{n}\right)=O\left(n^{-1 / 2}(\log \log n)^{1 / 2}\right)$.

From Theorems 1 and 2, it can be seen that the error of using GPS for approximation calculation is only related to $n$ and not to the dimensionality of the sample. Therefore, a suitable algorithm can be provided for the high-dimensional approximation calculation. Furthermore, from Theorem 3, the deviation of using GPS is $O\left(n^{-1+n}\right)$, while the deviation of using random method is $\mathrm{O}\left(n^{-1+n}\right)$. Therefore, using GPS to generate points is better than the random method.

\subsection{Algorithm for NSGA-III Multiobjective Optimization.}

Most of the multiobjective evolutionary algorithms are effective in solving problems with low objective dimensions. When the number of objectives is equal to or greater than 3 , which indicates the involvement of high-dimensional objective optimization problems, the increase in dimensions causes a decrease in selection pressure and results in unsatisfactory effects. In general, the frameworks of the elitist NSGA-III and NSGA-II algorithms are similar, and the only difference between them is the selection mechanism of individuals that are the offspring [41-44]. The NSGA-II algorithm selects individuals of the same nondominated level based on the calculated crowding distance, whereas the NSGA-III does so using a reference point-based method [45-47].

In the population initialization problem, although random initialization simplifies the complexity of the intelligence algorithm, it may make the initial distribution of the population uneven, leading the algorithm to fall into local optimum and affecting the convergence of the algorithm [48-50]. In order to solve this shortcoming, this paper adopts the GPS theory to initialize the population so that the population is uniformly distributed in the solution space to increase the diversity of the population and improve the algorithm's performance [51-53].

The GPS method and the random method are used to generate the two-dimensional initial population for comparison, as shown in Figures 2 and 3. With the same number of 600 points taken, the GPS method takes points more uniformly than the random method, and the population diversity is richer. Therefore, mapping the good points to the target solution space makes the initial population more ergodic, which can better achieve global optimization. 


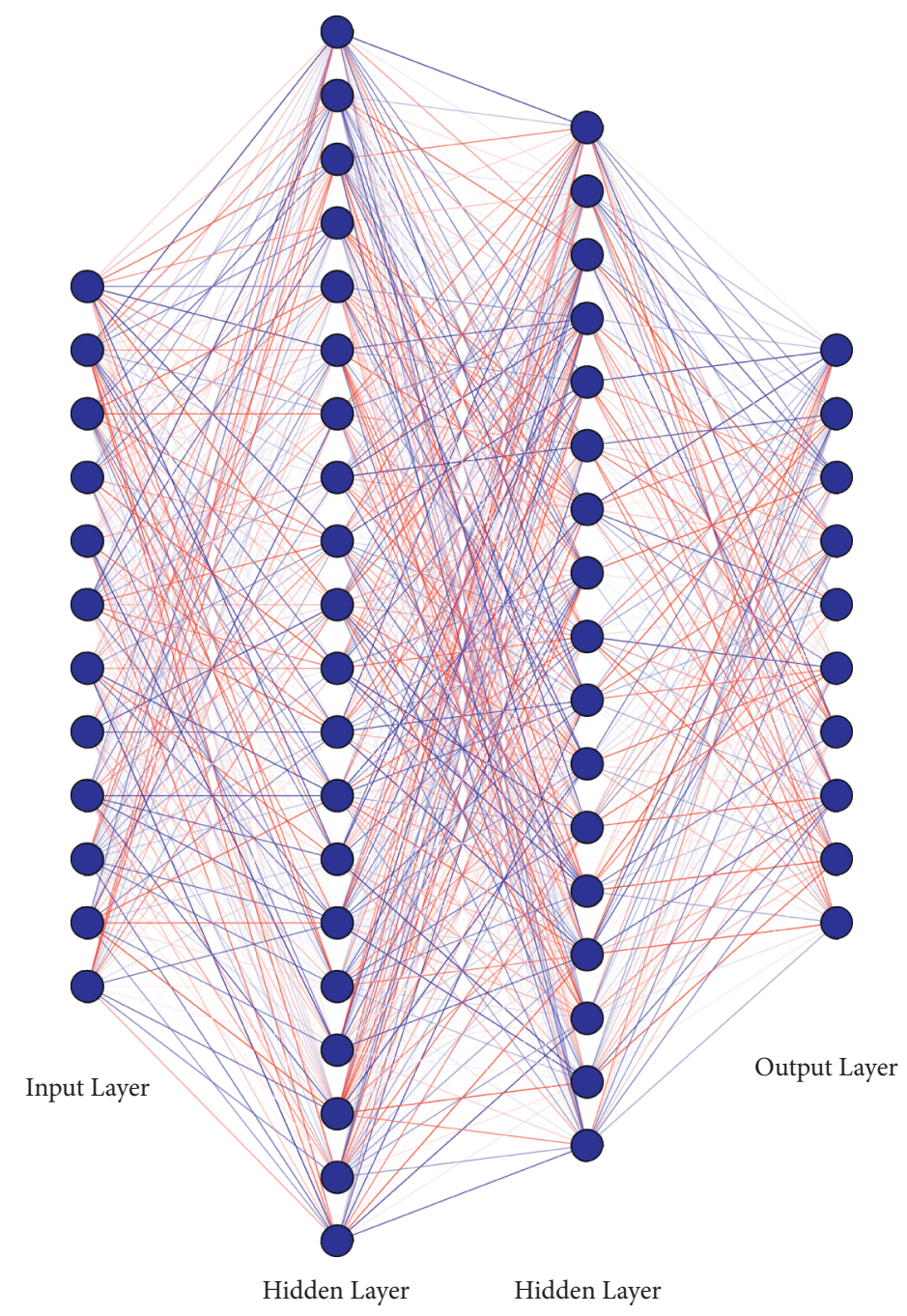

FIgURE 1: Structure of a NAR artificial neural network.

\section{Model Construction and Algorithm Design}

Storage space optimization involves multiple management decision-making objectives. A review of the literature by various scholars $[54,55]$ and the consideration of the requirements of business operations helped identify the objective functions of optimization as minimizing resource loss, maximizing goods accumulation, and minimizing goods selection time. They aimed to realize the rational use of resources and improve overall business performance.

\subsection{Model Construction}

4.1.1. Minimizing Resource Loss. The numbers of goods categories and storage spaces are similarly set to $n$; the different levels of order quantity are represented by $t ; T_{i}$ represents the $i^{\text {th }}$ type of order $(i=1,2, \ldots, t) ; f_{i}$ is the requested amount in the $i^{\text {th }}$ order; and dis $\left(T_{i}\right)$ represents the distance of the selection path for the $i^{\text {th }}$ order, where the start and end points of the forklift's selection path in the warehouse are the same. Further, a decision variable, $x_{j k}$, is added: product $j$ is on the goods shelf $k$ when $x_{j k}=1$ but not on $k$ when $x_{j k}=0$. This results in the objective function equation as follows:

$$
\begin{aligned}
& F_{1}=\min \sum_{i=1}^{m} f_{i} \operatorname{dis}\left(T_{i}\right)=\sum_{i=1}^{m} f_{i} \operatorname{dis}\left(x_{j k} j \in T_{i}, k=1,2, \ldots, n\right), \\
& \text { s.t. }\left\{\sum_{k=1}^{n} x_{j k}=1, j=1,2, \ldots, n, \sum_{j=1}^{m} x_{j k}=1, k=1,2, \ldots, n, x_{j k} \in\{0,1\}, \forall j, k .\right.
\end{aligned}
$$




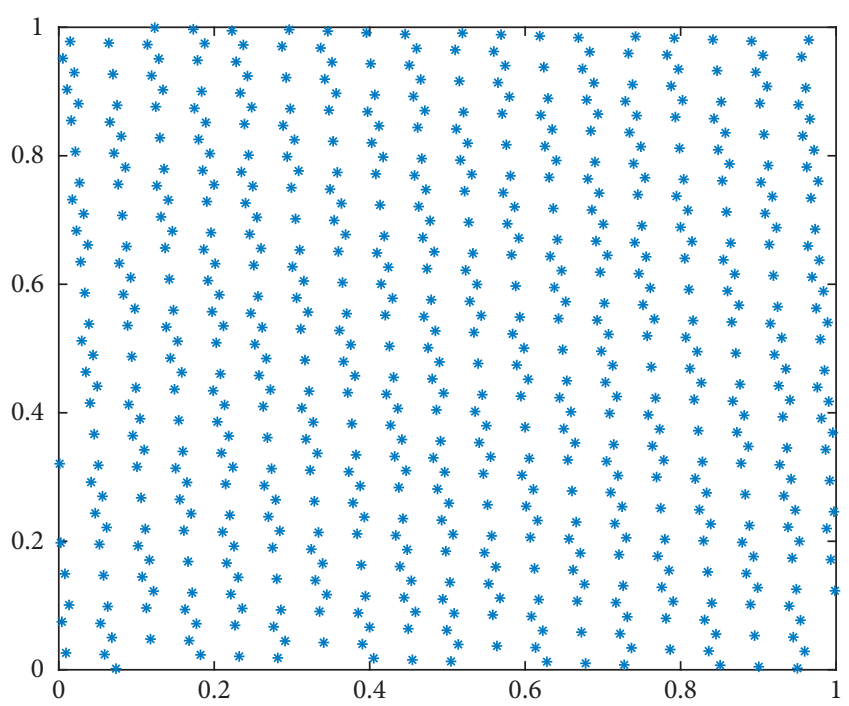

FIgURE 2: Two-dimensional initial populations generated by the good point set method $(N=600)$.

4.1.2. Maximizing Goods Accumulation. The accumulation level of different goods can be measured indirectly using the distance between goods. The storage location of the goods in a warehouse is indicated by $\left(x_{i j}, y_{i j}\right)$, the accumulation level of the same goods category isdi $s_{j}$, and the total accumulation level of goods in the warehouse is Dis. The equivalent coordinates of the same category of goods $\operatorname{are}\left(\overline{x_{i j}}, \overline{y_{i j}}\right)$, in which $\overline{x_{i j}}=\left(1 / n_{j}\right) \sum_{i=1}^{n_{j}}\left(x_{i j}-1\right), \overline{y_{i j}}=\left(1 / n_{j}\right)$ $\sum_{i=1}^{n_{j}}\left(y_{i j}-1\right)$; the equivalent coordinates of all the goods $\operatorname{are}(\bar{x}, \bar{y})$, in which $\bar{x}=(1 / n) \sum_{i=1}^{n} x_{i j}, \bar{y}=(1 / n) \sum_{i=1}^{n} y_{i j}$. This resulted in the objective function equation as follows [56]:

$$
\begin{aligned}
F_{2}(x, y) & =\max \left(\frac{\text { Dis }}{\sum_{j=1}^{n} \operatorname{dis}_{j}}\right), \\
\operatorname{dis}_{j} & =\sum_{i=1}^{n_{j}} \sqrt{\left(x_{i j}-\bar{x}_{j}-1\right)^{2}+\left(y_{i j}-\bar{y}_{j}-1\right)^{2}}, \\
\operatorname{Dis} & =\sum_{j=1}^{m} \sum_{i=1}^{n_{j}} \sqrt{\left(x_{i j}-\bar{x}-1\right)^{2}+\left(y_{i j}-\bar{y}-1\right)^{2}} .
\end{aligned}
$$

4.1.3. Minimizing Goods Selection Time. The time taken to complete the selection of one batch of goods was used to measure efficiency. The length and width of the goods shelf were set to $L$ and $H$, respectively, and the forklift's speed of lifting in the horizontal and vertical directions was $v_{x}$ and $v_{y}$, respectively. The time required for the forklift to select a single item from the goods shelf and reach the exit was represented by $t_{x y}$. Therefore, the objective function can be expressed as the following equation:

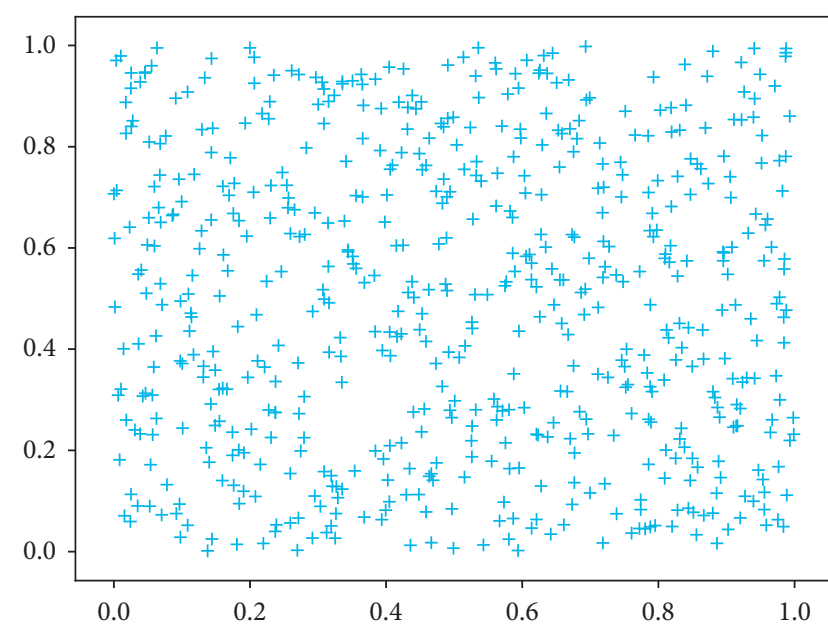

Figure 3: Two-dimensional initial populations generated by the random method $(N=600)$.

$$
\begin{aligned}
F_{3}(x, y) & =\min \sum_{x=1}^{m} \sum_{y=1}^{n} t_{x y}, \\
t_{x y} & =\max \left\{(x-1) \times \frac{L}{v_{x}},(y-1) \times \frac{H}{v_{y}}\right\} .
\end{aligned}
$$

4.2. Steps of the Algorithm. It is necessary to predict future demand to realize highly responsive warehousing operations, and, in general, the required resources and equipment are reasonably allocated before the arrival of order requests. Reasonable predictions of order requests can be used as an effective data source for subsequent warehouse storage space allocation as well. By inputting predicted demand into a multiobjective optimization model, one can determine the location of goods expected to be included in customers' future requests. Figure 4 depicts the steps of the storage space algorithm.

The algorithm was executed in three stages. The processing mechanism of each stage is described here. Stage 1 involved data preprocessing and demand prediction. The first step was to examine data regularity. Ideally, the data satisfy the criterion; that is, the records are complete, and format requirements are satisfied. In this case, the dataset was directly segregated and input into the NAR neural network for prediction [57]. If the requirements were not satisfied, the data were transferred to the data preprocessing phase, in which the algorithm activated data processing methods, such as missing data supplementation, outlier processing, and data noise reduction [58]. When the preprocessed data satisfied the neural network's requirements, the data were input into the network to make predictions.

Stage 2 involved the error testing of predicted results. The algorithm evaluated the prediction effect using the RMSE, MSE, and coefficients of autocorrelated errors [59-61]. If the error in prediction was within the acceptable 
range, the predicted results of the data were used as inputs for the next stage of storage space allocation. Otherwise, the algorithm's feedback mechanism was activated, and the predicted effects were continuously relayed into the network until the prediction error became lower than the error threshold.

Stage 3 facilitated the allocation of storage space, which was oriented toward the predicted demand. When the error in predicted results was within the acceptable range, the next phase ensured the allocation of storage space. Then, the mathematical objectives and function models that needed to be optimized were self-defined, the data were checked, and the iterative process to find a solution was initiated. The NSGA-III continued seeking solutions until the results of the allocation of storage space for goods satisfied the predefined solution plane and realized the Pareto optimal value [62]. Finally, the algorithm output the results of the solution, which could serve as a reference for decision-makers.

\section{Data Calculation Example}

5.1. Data Preprocessing. The case study data used in this study were obtained from Kaggle, a renowned data science competition platform. The dataset contained the historical product demand of a manufacturing company with footprints worldwide and included 2,172 product stock keeping units in 33 product categories distributed in four warehouses. Following data preprocessing, the time-series demand data of each product were sorted, and a case study examination was conducted on each product's time-series demand data. One product was used as an example to predict an order request. Subsequently, the same process was applied to build the algorithm for the orders of other products.

First, the data were downsampled, and the data resampling technique was used to convert the data from the original to another frequency. The process of data conversion from a high to a low frequency is defined as downsampling [63]. The original demand data of warehouse goods were recorded according to the daily demand for e-commerce goods. However, during actual warehouse operations, warehouse management personnel usually use nonrecording methods for goods with a multiday demand of 0 . These unrecorded data affect data quality during data processing. Hence, for the purpose of processing, downsampling was used to convert the data based on the frequency of weekly statistics.

Figure 5 indicates that the records in the original dataset were dense. The data for weekly demand were aggregated after downsampling, which effectively eliminated the effect of missing values that were not recorded when the goods demand data were 0, as shown in Figure 6. The processes of preparing data and exploring fitting models are easier in the case of smaller datasets. For warehousing operations, one week is a reasonable and complete operational cycle. The original data contained substantial noise, which was caused by the use of inefficient recording and measurement methods and the impact of mutation factors. Such noise in the data made the model fitting difficult or increased the costs associated with fitting. In this study, the logarithm for the original data, $t s \prime=\log (t s)$, was calculated and input into the model for training. As shown in Figure 7, after the logarithmic transformation process, the data series congregated within a certain range and fluctuated steadily, whereas the original data had substantial fluctuations.

The next step was to train, verify, and test the accuracy of the prediction model from Stage 1 onward. For this purpose, the algorithm segmented the data of goods demand into the training, verification, and test sets in the ratio of 70\%:25\%: 5\%. In Figure 8, the blue, orange, and green lines represent the data of the training, verification, and test sets, respectively. These datasets contained a total of 183 samples, 65 statistical data records, and 13 segments. The green line is applicable to a later stage and measures prediction accuracy.

\subsection{Multiobjective Optimization of Storage Space Allocation.} The 183 time-series data records were used as the training set; 65 data records were used as the verification set; and remaining 13 goods circulation data records were input into the algorithm as the test set for data fitting, verification, and prediction. The cyan line in Figure 9 represents the model's value for predicted demand based on the existing feature values, whereas the blue line represents the actual demand in the test set. The model had a prediction accuracy rate of $98.79 \%$.

The data pertaining to future demand were input into the multiobjective storage space optimization model to obtain storage space allocation results. The maximum number of evolutions that we set in our study was 400 , and the evolution operator was simulated binary crossover with polynomial mutation. The crossover rate was set to 0.9 , and the variance is the inverse of the dimensionality of the decision variables. The set in the solution plane of the elitist NSGA was a noninferior solution that satisfied the Pareto optimal law [64], and we examined all of the solution sets in the solution plane.

5.3. Comparison and Verification of Methods. The results of three prediction models-NAR, the prophet time-series prediction framework [65-67], and linear regression-were compared, and the prediction effect of the algorithm's Stage 1 was evaluated. Figure 10 depicts the results. Among the tested methods, the NAR prediction results were the closest to the actual goods demand, and its prediction accuracy rate was the highest.

Subsequently, the following methods were used to verify the effectiveness of the NAR-NSGA-III storage space allocation algorithm: random allocation, $\mathrm{ABC}$ classification, and the simulation of the multiobjective storage space allocation algorithm based on demand prediction [68]. Specifically, the goods selection efficiencies of the various schemes were verified using the goods selection time during operations [69]. Among them, the random allocation scheme did not comply with the optimization principle and caused the random placement of goods on storage shelves. On the other hand, the $\mathrm{ABC}$ classification method first classified the importance of goods according to their historical demand data and then randomly allocated goods in the same category. 


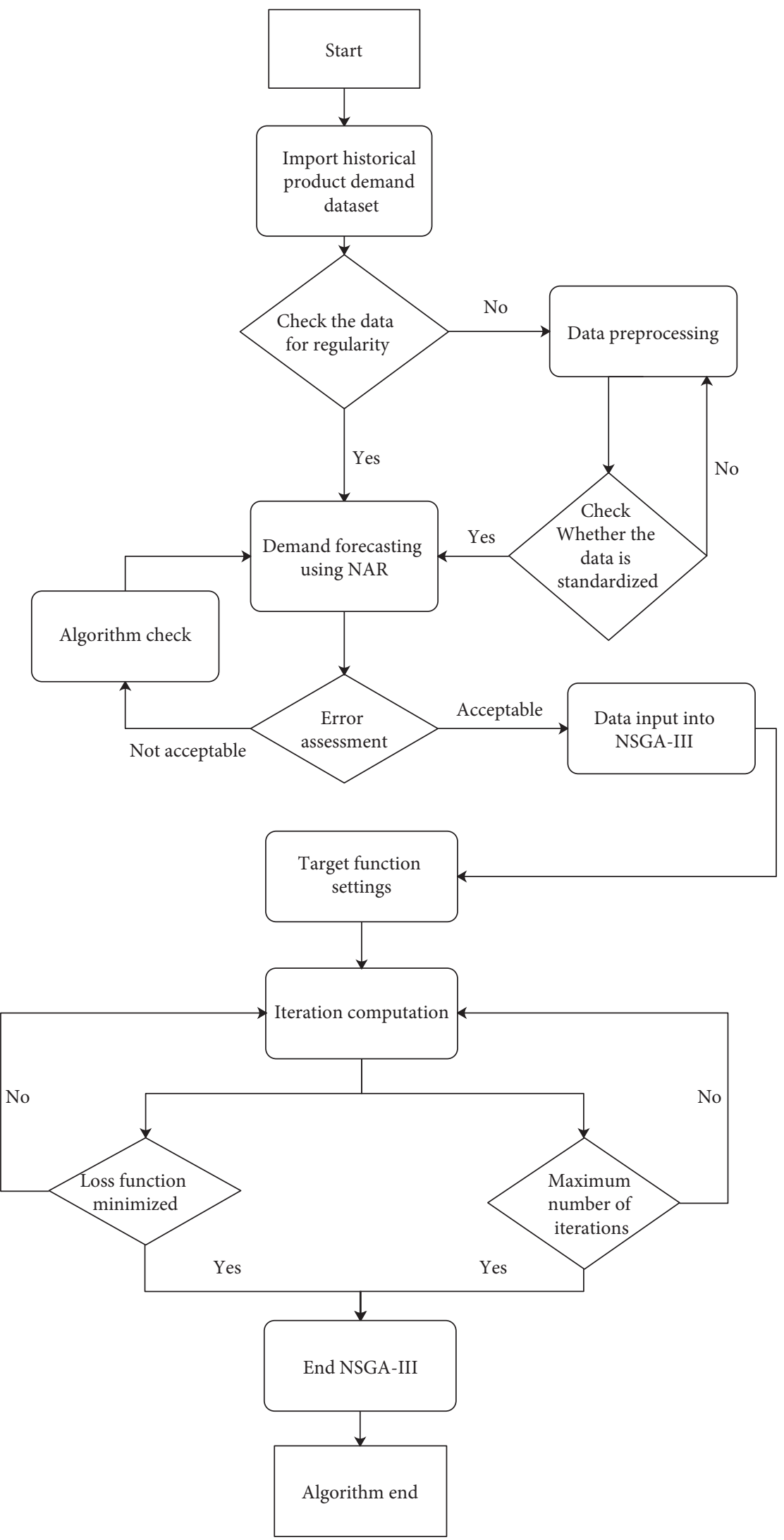

FIGURE 4: Steps of the storage space allocation algorithm based on nonlinear autoregressive neural network and nondominated sorting genetic algorithm III. 


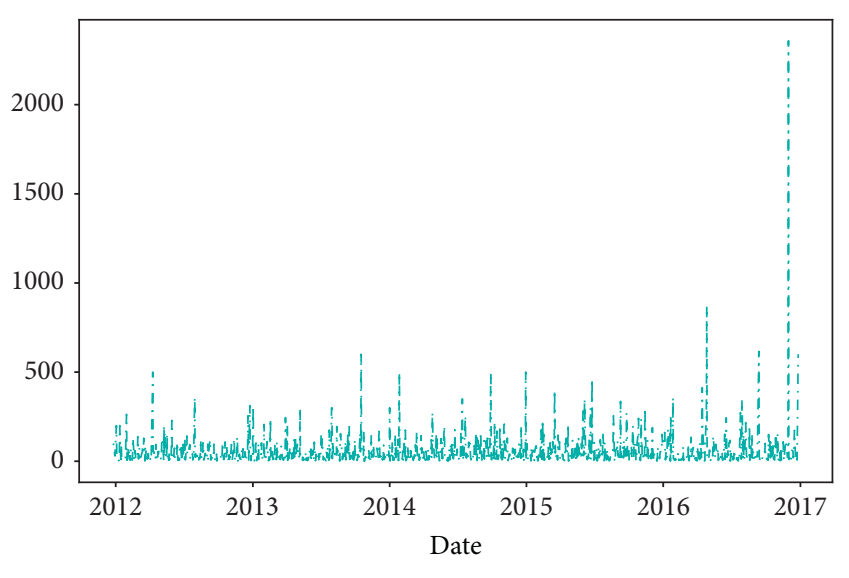

-..- Order Demand

Figure 5: Distribution of demand data for original goods.

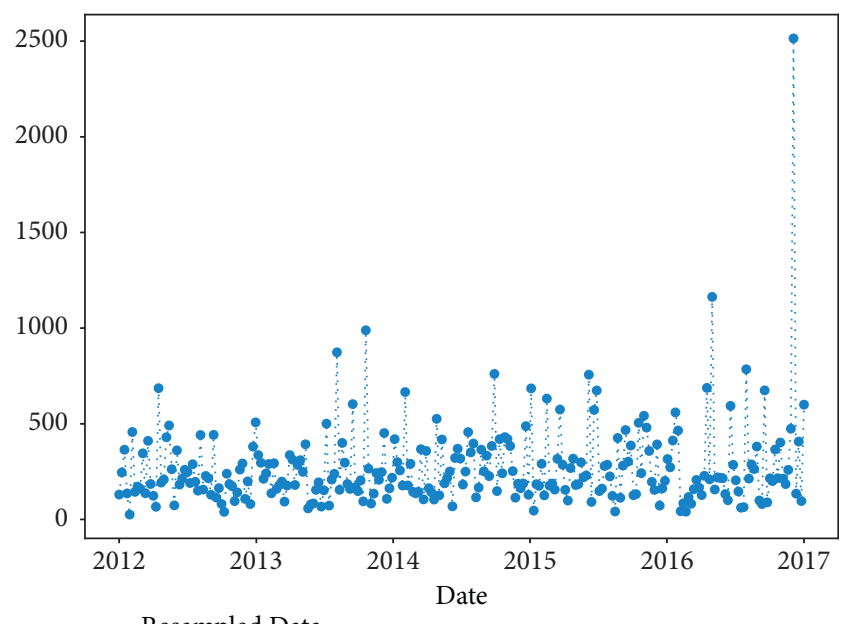

-.. Resampled Data

FIGURE 6: Distribution of downsampled demand data.

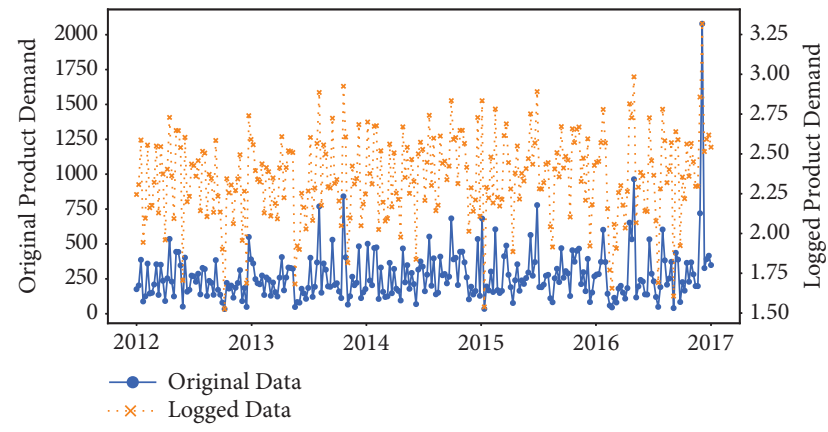

Figure 7: Comparison of the raw data before and after logarithmic processing.

The three storage space allocation schemes were run 20 times each, and the final goods selection efficiency was determined by calculating the average efficiency of the 20 selection times. The simulation results, as shown in Figure 11, indicate that the allocation scheme based on NAR and NSGAIII improved goods selection efficiency. The completion time for 20 runs of the NAR-NSGA-III allocation scheme was less

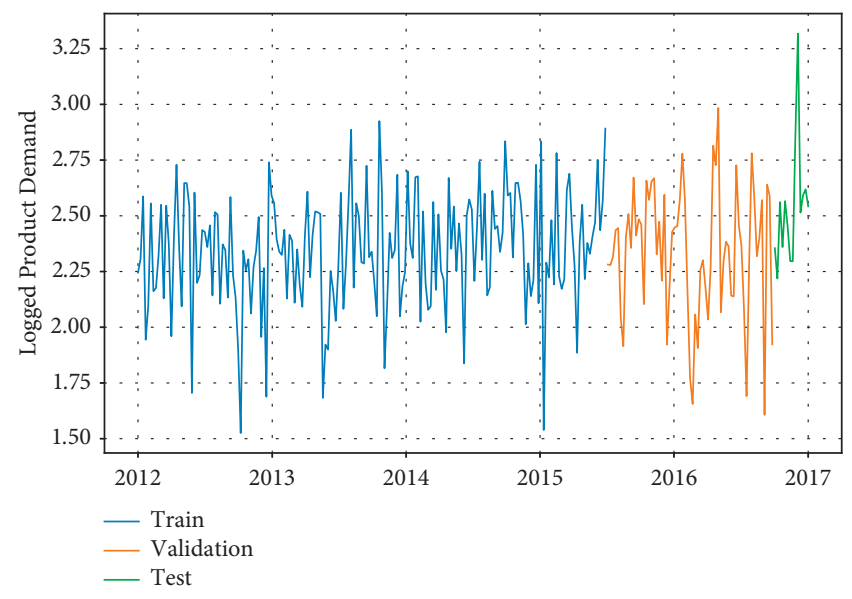

Figure 8: Segmented line graph showing various datasets.

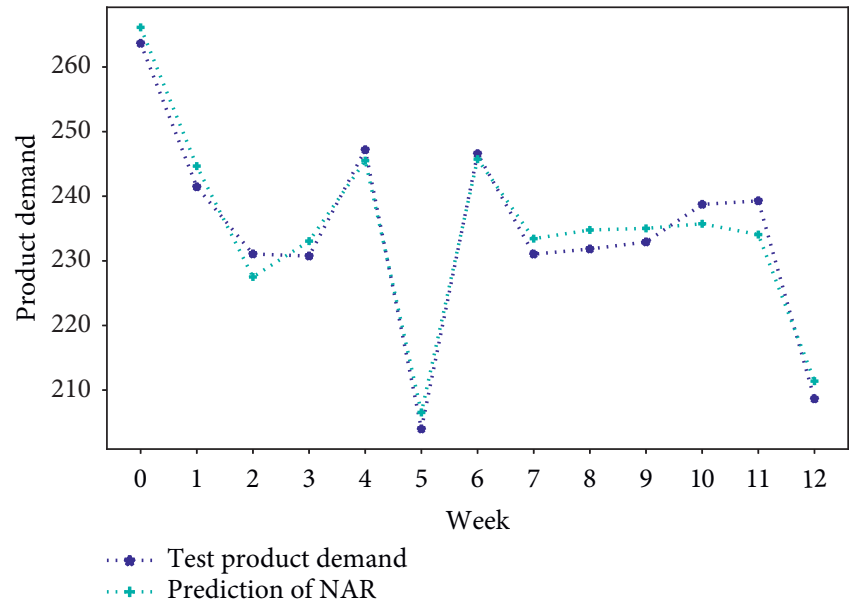

Figure 9: NAR predicted and actual values.

than the times of the other two allocation schemes, and the goods selection time was reduced by as much as $23.8 \%$. After considering the optimization objectives such as accumulation of goods and resource loss, NAR-NSGA-III's cargo space allocation strategy facilitates the fast response of picking process in the simulation environment, thus achieving superior picking efficiency.

\section{Discussion}

The algorithm not only provided a mechanism for efficient data processing but also identified the optimal decision point under multiple complex objectives. It compensated for the shortcomings of current theoretical research, which lacks comprehensiveness. The proposed algorithm can be integrated into management practice as well: the task of predicting future product demand can be completed by connecting to the enterprise's database, then reading a large amount of data before conducting a series of data analyses and running the algorithm's internal processing mechanism $[70,71]$. When changes in product demand are updated and synchronized in real time, the effect of real-time prediction and allocation of goods can be achieved as well. 


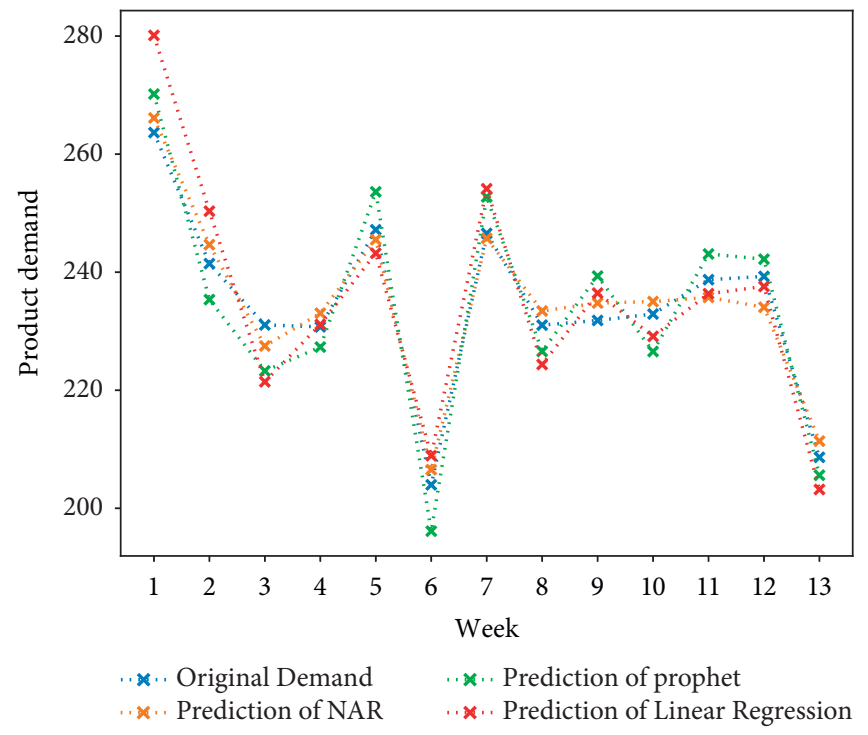

Figure 10: Results of different prediction algorithms.

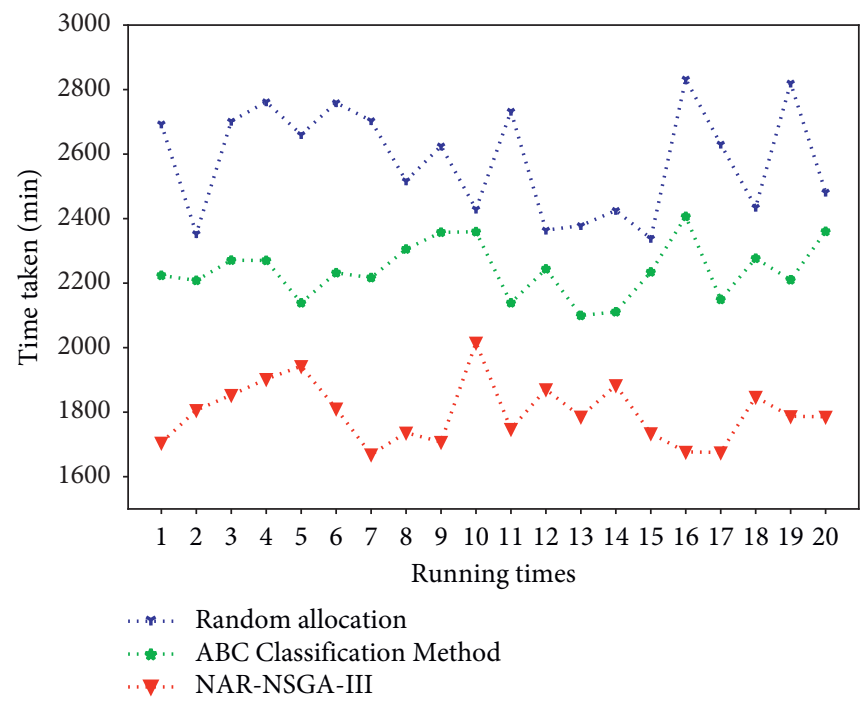

FIGURE 11: Line graph comparing goods selection times.

This study was the first to combine the problems of order demand forecasting and multiobjective space allocation, giving reference to both order management and space allocation for warehouses. Nevertheless, the methods used in this study are not diverse enough, and there are few accuracy measures for the prediction results [72, 73]. Future research will analyze the initial reference point size setting and expand the reference point screening strategy to make the retained reference points with better distribution. The next step should also discuss the determination of evolutionary stages and adopt more reasonable thresholds to make the stage classification more accurate [74-76].

\section{Conclusions}

The findings of this study provided theoretical support for e-commerce distribution centers to develop storage space allocation strategies in big data processing contexts. The NAR artificial neural network and NSGA-III multiobjective optimization algorithm were used to construct a data-driven model algorithm to optimize storage space allocation. Simulations were used for verification and, compared to other methods, the storage space allocation method proposed by this study enabled the reduction of selection time for the same batch of goods by up to $23.8 \%$.

The study's conclusion can serve as an important reference for e-commerce warehouses to predict order requests and allocate storage space. The logistics industry has suffered numerous losses due to unreasonable demand management and storage space allocation, and this study meets the needs of the logistics industry and society as a whole. By reducing the unreasonable use of resources by logistics enterprises, the industry's overall operational efficiency will be improved, and society will thus achieve sustainable development. 


\section{Data Availability}

Data can be obtained from the Kaggle official website.

\section{Conflicts of Interest}

The authors declare that they have no conflicts of interest.

\section{Authors' Contributions}

Peijian Wu has managed supervision, reviewing, and editing. Yulu Chen has finished literature review, methodology, results, and writing.

\section{References}

[1] J. Saderova, A. Rosova, M. Sofranko, and P. Kacmary, "Example of warehouse system design based on the principle of logistics," Sustainability, vol. 13, no. 8, APR 2021.

[2] Yi-hua Chen, "Intelligent algorithms for cold chain logistics distribution optimization based on big data cloud computing analysis," Journal of Cloud Computing-Advances Systems and Applications, vol. 9, no. 1, JUL 132020.

[3] Y. Zhao, Y. Zhou, and W. Deng, "Innovation mode and optimization strategy of B2C E-commerce logistics distribution under big data," Sustainability, vol. 12, no. 8, APR 2020.

[4] M. R. Islam, S. M. Ali, . M. Fathollahi-Fard, and G. Kabir, “A novel particle swarm optimization-based grey model for the prediction of warehouse performance," Journal Of Computational Design And Engineering, vol. 8, no. 2, pp. 705-727, APR 2021.

[5] L. De Armas, D. Valdes, C. Morell, and R. Bello, "Solutions to storage spaces allocation problem for import containers by exact and heuristic methods," Computación Y Sistemas, vol. 23, no. 1, pp. 197-211, 2019.

[6] J. Gu, M. Goetschalckx, and L. F. McGinnis, "Solving the forward-reserve allocation problem in warehouse order picking systems," Journal of the Operational Research Society, vol. 61, no. 6, pp. 1013-1021, JUN 2010.

[7] F. T. S. Chan and H. K. Chan, "Improving the productivity of order picking of a manual-pick and multi-level rack distribution warehouse through the implementation of class-based storage," Expert Systems with Applications, vol. 38, no. 3, pp. 2686-2700, MAR 2011.

[8] J. Li, M. Moghaddam, and S. Y. Nof, "Dynamic storage assignment with product affinity and $\mathrm{ABC}$ classification-a case study," International Journal of Advanced Manufacturing Technology, vol. 84, no. 9-12, JUN 2016.

[9] N. Zaerpour, B. Rene, M. de Koster, and Y. Yu, "Storage policies and optimal shape of a storage system," International Journal of Production Research, vol. 51, no. 23-24, pp. 68916899, NOV 12013.

[10] B. Sundarakani, A. Ajaykumar, and A. Gunasekaran, "Big data driven supply chain design and applications for blockchain: an action research using case study approach \& nbsp," Omega-International Journal of Management Science, vol. 102, JUL 2021.

[11] B. Zhang, R. Tan, and C.-J. Lin, "Forecasting of e-commerce transaction volume using a hybrid of extreme learning machine and improved moth-flame optimization algorithm," Applied Intelligence, vol. 51, no. 2, pp. 952-965, 2021.

[12] M. Kizil, "A holistic approach for the problem of storage space allocation in cellular manufacturing," International Journal of
Advanced Manufacturing Technology, vol. 48, no. 9-12, pp. 1031-1043, 2010.

[13] S. S. Rao and G. K. Adil, "Class-based storage assignment in a unit-load warehouse employing as/rs with inventory space allocation considering product specific setup to holding cost ratio," Asia Pacific Journal of Operational Research, vol. 31, no. 5, Article ID 1450034, 2014.

[14] B. Y. Ekren, "A multi-objective optimisation study for the design of an AVS/RS warehouse," International Journal of Production Research, vol. 59, no. 4, pp. 1107-1126, FEB 16 2021.

[15] Y. Liu, S. Ji, Z. Su, and D. Guo, "Multi-objective AGV scheduling in an automatic sorting system of an unmanned (intelligent) warehouse by using two adaptive genetic algorithms and a multi-adaptive genetic algorithm," PLoS One, vol. 14, no. 12, DEC 62019.

[16] S. Quintanilla, A. Perez, F. Ballestin, and P. Lino, "Heuristic algorithms for a storage location assignment problem in a chaotic warehouse," Engineering Optimization, vol. 47, no. 10, pp. 1405-1422, OCT 32015.

[17] F. Ramtin and J. A. Pazour, "Product allocation problem for an AS/RS with multiple in-the-aisle pick positions," IIE Transactions, vol. 47, no. 12, pp. 1379-1396, DEC 22015.

[18] M. Ang, Y. F. Lim, and M. Sim, "Robust storage assignment in unit-load warehouses," Management Science, vol. 58, no. 11, pp. 2114-2130, NOV 2012.

[19] D. M.-H. Chiang, C.-P. Lin, and M.-C. Chen, "The adaptive approach for storage assignment by mining data of warehouse management system for distribution centres," Enterprise Information Systems, vol. 5, no. 2, pp. 219-234, 2011.

[20] J. A. Larco, R. De Koster, K. Jan Roodbergen, and D. Jan, "Managing warehouse efficiency and worker discomfort through enhanced storage assignment decisions," International Journal of Production Research, vol. 55, no. 21, pp. 6407-6422, 2017.

[21] P. Bodnar and J. Lysgaard, "A dynamic programming algorithm for the space allocation and aisle positioning problem," Journal of the Operational Research Society, vol. 65, no. 9, pp. 1315-1324, SEP 2014.

[22] S. Ene, I. Kucukoglu, A. Aksoy, and N. Ozturk, "A genetic algorithm for minimizing energy consumption in warehouses," Energy, vol. 114, pp. 973-980, NOV 12016.

[23] H. J. Carlo and G. E. Giraldo, "Toward perpetually organized unit-load warehouses," Computers \& Industrial Engineering, vol. 63, no. 4, pp. 1003-1012, DEC 2012.

[24] J. C.-H. Pan, Po-H. Shih, M.-H. Wu, and J.-H. Lin, “A storage assignment heuristic method based on genetic algorithm for a pick-and-pass warehousing system," Computers \& Industrial Engineering, vol. 81, no. 1-13, MAR 2015.

[25] O. Cruz-Dominguez and R. Santos-Mayorga, "Artificial intelligence applied to assigned merchandise location IN retail sales systems," South African Journal of Industrial Engineering, vol. 27, no. 1, pp. 112-124, MAY 2016.

[26] B. S. Kim and J. S. Smith, "Slotting methodology using correlated improvement for a zone-based carton picking distribution system," Computers \& Industrial Engineering, vol. 62, no. 1, pp. 286-295, FEB 2012.

[27] D. Arunachalam, N. Kumar, and J. P. Kawalek, "Understanding big data analytics capabilities in supply chain management: unravelling the issues, challenges and implications for practice," Transportation Research Part E: Logistics and Transportation Review, vol. 114, pp. 416-436, JUN 2018.

[28] E. d. R. Francisco, J. L. Kugler, S. M. Kang, R. Silva, and P. A. Whigham, "Beyond technology: management challenges 
IN the big data era," Rae-Revista De Administracao De Empresas, vol. 59, no. 6, pp. 375-378, NOV-DEC 2019.

[29] D. Mezzogori and F. Zammori, "An entity embeddings deep learning approach for demand forecast of highly differentiated products," Procedia Manufacturing, vol. 39, pp. 1793$1800,2019$.

[30] J. Huber and H. Stuckenschmidt, "Daily retail demand forecasting using machine learning with emphasis on calendric special days," International Journal of Forecasting, vol. 36, no. 4, pp. 1420-1438, OCT-DEC 2020.

[31] S. Xu and H. K. Chan, "Forecasting medical device demand with online search queries: a big data and machine learning approach," Procedia Manufacturing, vol. 39, pp. 32-39, 2019.

[32] E. Tarallo, G. K. Akabane, C. I. Shimabukuro, J. Mello, and A. Douglas, "Machine learning in predicting demand for fastmoving consumer goods: an exploratory research," IFACPapersOnLine, vol. 52, no. 13, pp. 737-742, 2019.

[33] M. E. Fontana and C. A. V. Cavalcante, "Using the efficient frontier to obtain the best solution for the storage location assignment problem," Mathematical Problems in Engineering, vol. 2014, Article ID 745196, 10 pages, 2014.

[34] J.-P. Gagliardi, J. Renaud, and A. Ruiz, "On storage assignment policies for unit-load automated storage and retrieval systems," International Journal of Production Research, vol. 50, no. 3, pp. 879-892, 2012.

[35] E. Raveh, Y. Ofek, B. Ron, and H. Cohen, "Applying Big Data visualization to detect trends in 30 years of performance reports," Evaluation, vol. 26, no. 4, pp. 516-540, OCT 2020.

[36] N. M. Watanabe, S. Shapiro, and J. Drayer, "Big data and analytics in sport management," Journal of Sport Management, vol. 35, no. 3, MAY 2021.

[37] S. Kumar, R. Kumar, and Sunayana, "Forecasting of municipal solid waste generation using non-linear autoregressive (nar) neural models," Waste Management, vol. 121, pp. 206-214, 2021.

[38] R. Sarkar, S. Julai, S. Hossain, W. T. Chong, and M. Rahman, "A comparative study of activation functions of NAR and NARX neural network for long-term wind speed forecasting in Malaysia," Mathematical Problems in Engineering, vol. 2019, Article ID 6403081, 14 pages, 2019.

[39] M. Adil, R. Ullah, S. Noor, and N. Gohar, "Effect of number of neurons and layers in an artificial neural network for generalized concrete mix design," Neural Computing \& Applications, pp. 1-9, 2020.

[40] S. Wei, D. Zuo, and J. Song, "Improving prediction accuracy of river discharge time series using a wavelet-nar artificial neural network," Journal of Hydroinformatics, vol. 14, no. 4, pp. 974-991, 2012.

[41] M. R. Bagheri, M. Mosayebi, A. Mandian, and K. Ahmad, "Weighted sum Pareto optimization of a three dimensional passenger vehicle suspension model using NSGA-II for ride comfort and ride safety," Smart Structures and Systems, vol. 22, no. 4, pp. 469-479, OCT 2018.

[42] H. Jain and K. Deb, "An evolutionary many-objective optimization algorithm using reference-point based nondominated sorting approach, part ii: handling constraints and extending to an adaptive approach," IEEE Transactions on Evolutionary Computation, vol. 18, no. 4, pp. 602-622, 2013.

[43] E. Ozahi and A. Tozlu, "Optimization of an adapted Kalina cycle to an actual municipal solid waste power plant by using NSGA-II method," Renewable Energy, vol. 149, pp. 11461156, APR 2020.

[44] N. Vural, Z. Y. Hitit, and S. Ertunç, "Multi-objective optimization of drying conditions for the olea europaea l. leaves with NSGA-II," Journal of Food Processing and Preservation, vol. 45, Article ID e15625, 2021.

[45] K. Deb and H. Jain, "An evolutionary many-objective optimization algorithm using reference-point-based nondominated sorting approach, part i: solving problems with box constraints," IEEE Transactions on Evolutionary Computation, vol. 18, no. 4, pp. 577-601, 2013.

[46] N. H. Truong and D.-N. Dao, "New hybrid between NSGA-III with multi-objective particle swarm optimization to multiobjective robust optimization design for Powertrain mount system of electric vehicles," Advances in Mechanical Engineering, vol. 12, no. 2, FEB 2020.

[47] A. M. Usman, U. K. Yusof, and S. Naim, "Filter-based multiobjective feature selection using NSGA III and cuckoo optimization algorithm," IEEE ACCESS, vol. 8, 2020.

[48] T. Chang, D. Kong, H. Na, K. Xu, and G. Yang, "Solving the dynamic weapon target assignment problem by an improved artificial bee colony algorithm with heuristic factor initialization," Applied Soft Computing, vol. 70, pp. 845-863, SEP 2018.

[49] T. Maini, A. Kumar, R. K. Misra, and D. Singh, "Intelligent fuzzy rough set based feature selection using swarm algorithms with improved initialization," Journal of Intelligent and Fuzzy Systems, vol. 37, no. 1, pp. 1155-1164, 2019.

[50] I. Vlasic, M. Durasevic, and D. Jakobovic, "Improving genetic algorithm performance by population initialisation with dispatching rules," Computers \& Industrial Engineering, vol. 137, NOV 2019.

[51] Y. Cao, H. Yan, T. Liu, and J. Yang, "Application of quasiMonte Carlo method based on good point set in tolerance analysis," Journal of Computing and Information Science in Engineering, vol. 16, no. 2, JUN 2016.

[52] Y. Li, Z. Ni, F. Jin, J. Li, and F. Li, "Research on clustering method of improved glowworm algorithm based on goodpoint set," Mathematical Problems in Engineering, vol. 2018, Article ID 8724084, 8 pages, 2018.

[53] F. Wang, Y. Zhang, and L. Ma, "Modeling and analysis of a self-learning worm based on good point set scanning," Wireless Communications and Mobile Computing, vol. 9, no. 4, pp. 573-586, APR 2009.

[54] Z. K. Hou, "The optimization of automated goods dynamic allocation and warehousing model," Computer Optics, vol. 44, no. 5, 2020.

[55] D. Yang, Y. Wu, and W. Ma, "Optimization of storage location assignment in automated warehouse," Microprocessors and Microsystems, vol. 80, Article ID 103356, 2021.

[56] Y.-L. Jiao, X.-c. Xing, P. Zhang, L.-C. Xu, and X.-R. Liu, "Multi-objective storage location allocation optimization and simulation analysis of automated warehouse based on multipopulation genetic algorithm," Concurrent Engineering, vol. 26, no. 4, pp. 367-377, 2018.

[57] K. Rahul and R. Kumar Banyal, "Detection and correction of abnormal data with optimized dirty data: a new data cleaning model," International Journal of Information Technology and Decision Making, vol. 20, no. 2, pp. 809-841, MAR 2021.

[58] A. H. Adineh, Z. Narimani, and S. Chandra Satapathy, "Importance of data preprocessing in time series prediction using sarima: a case study," International Journal of Knowledge-Based and Intelligent Engineering Systems, vol. 24, no. 4, pp. 331-342, 2020.

[59] A. H. Bukhari, M. Sulaiman, M. A. Z. Raja, M. A. Z. Raja, S. Islam, and M. Shoaib, "Design of a hybrid nar-rbfs neural network for nonlinear dusty plasma system," Alexandria Engineering Journal, vol. 59, no. 5, pp. 3325-3345, 2020. 
[60] A. de Ramon-Fernandez, M. J. Salar-Garcia, D. Ruiz Fernandez, J. Greenman, and I. A. Ieropoulos, "Evaluation of artificial neural network algorithms for predicting the effect of the urine flow rate on the power performance of microbial fuel cells," Energy, vol. 213, DEC 152020.

[61] Y. Qiu, J. Zhou, M. Khandelwal, H. Yang, P. Yang, and C. Li, "Performance evaluation of hybrid woa-xgboost, gwoxgboost and bo-xgboost models to predict blast-induced ground vibration," Engineering with Computers, pp. 1-18, 2021.

[62] Y. Hou, N. Q. Wu, Z. W. Li, Y. Zhang, T. Qu, and Q. H. Zhu, "Many-objective optimization for scheduling of crude oil operations based on nsga- with consideration of energy efficiency," Swarm and Evolutionary Computation, vol. 57, Article ID 100714, 2020.

[63] H. Singh Shekhawat and G. Meinsma, "A sampled-data approach to optimal non-causal downsampling," Mathematics of Control, Signals, and Systems, vol. 27, no. 3, pp. 277-315, 2015.

[64] X. Sun, Y. Wang, H. Kang, Y. Shen, Q. Chen, and D. Wang, "Modified multi-crossover operator nsga-iii for solving low carbon flexible job shop scheduling problem," Processes, vol. 9, no. 1, p. 62, 2021.

[65] M. Navratil and A. Kolkova, "Decomposition and forecasting time series in the business economy using prophet forecasting model," Central European Business Review, vol. 8, no. 4, p. 26, 2019.

[66] M. Vetri Selvi and S. Mishra, "Investigation of performance of electric load power forecasting in multiple time horizons with new architecture realized in multivariate linear regression and feed-forward neural network techniques," IEEE Transactions on Industry Applications, vol. 56, no. 5, pp. 5603-5612, SEPOCT 2020.

[67] P. Theerthagiri, "Forecasting hyponatremia in hospitalized patients using multilayer perceptron and multivariate linear regression techniques," Concurrency and Computation: Practice and Experience, vol. 33, Mar 2021.

[68] T. Franzke, E. H. Grosse, C. H. Glock, and R. Elbert, "An investigation of the effects of storage assignment and picker routing on the occurrence of picker blocking in manual picker-to-parts warehouses," International Journal of Logistics Management, vol. 28, no. 3, pp. 841-863, 2017.

[69] Y. R. Wang and A. N. Chen, "Production logistics simulation and optimization of industrial enterprise based on flexsim," International Journal of Simulation Modelling, vol. 15, no. 4, pp. 732-741, 2016.

[70] C. Chen, C. Hou, J. Xiao, Y. Wen, and X. Yuan, "Enhancing purchase behavior prediction with temporally popular items," IEICE - Transactions on Info and Systems, vol. E100D, no. 9, pp. 2237-2240, SEP 2017.

[71] L. F. Sales, A. Pereira, T. Vieira, and E. de Barros Costa, "Multimodal deep neural networks for attribute prediction and applications to e-commerce catalogs enhancement," Multimedia Tools and Applications, vol. 80, no. 17, pp. 25851-25873, JUL 2021.

[72] M. O. Elish, "A comparative study of fault density prediction in aspect-oriented systems using MLP, RBF, KNN, RT, DENFIS and SVR models," Artificial Intelligence Review, vol. 42, no. 4, pp. 695-703, DEC 2014.

[73] X. Yuan, C. Chen, X. Lei, Y. Yuan, and M. A. Rana, "Monthly runoff forecasting based on LSTM-ALO model," Stochastic Environmental Research and Risk Assessment, vol. 32, no. 8, pp. 2199-2212, AUG 2018.
[74] W. Deng, S. Shang, X. Cai, H. Zhao, Y. Song, and J. Xu, “An improved differential evolution algorithm and its application in optimization problem," Soft Computing, vol. 25, no. 7, pp. 5277-5298, APR 2021.

[75] X. Ran, X. Zhou, M. Lei, W. Tepsan, and W. Deng, "A novel k-means clustering algorithm with a noise algorithm for capturing urban hotspots," Applied Sciences, vol. 11, no. 23, 2021.

[76] H. Shao, J. Lin, L. Zhang, D. Galar, and U. Kumar, "A novel approach of multisensory fusion to collaborative fault diagnosis in maintenance," Information Fusion, vol. 74, pp. 65-76, 2021. 